\title{
Psicooncología
}

ISSN: 1696-7240

http://dx.doi.org/10.5209/PSIC.55816

\section{Escala de estrategias de enfrentamiento para cuidadores primarios informales de pacientes con cáncer}

\author{
Fatima Sagrario Espinoza-Salgado ${ }^{1 *}$; Isabel Reyes-Lagunes²; Eduardo Velasco-Rojano ${ }^{3}$; \\ María Cristina Bravo-González ${ }^{4}$; Armando Ramírez Ramírez ${ }^{5}$
}

Recibido: 24 de enero de 2017 / Aceptado: 15 de abril de 2017

Resumen. Objetivo: Analizar propiedades psicométricas de un nuevo instrumento válido y confiable, que evalúa las estrategias de enfrentamiento en cuidadores primarios informales (CPI) de pacientes oncológicos. El Instrumento de Estrategias de Enfrentamiento en Cuidadores Primarios Informales (IEECPI), consta de 2 factores (Dirigido a la emoción) y 3 indicadores (Dirigido al problema). Método: Se evaluó a 20 CPI de pacientes oncológicos mediante un cuestionario de preguntas abiertas, para obtener información sobre la conceptualización de las dimensiones teóricas de las estrategias de enfrentamiento obteniendo 216 indicadores, agrupados en dos categorías (Dirigido a la emoción y al problema), de los cuales se generaron 55 reactivos que se aplicaron a 255 CPI. Una vez obtenidos los datos se siguió el procedimiento propuesto por Nunnally y Bernstein (1994) y el de Reyes-Lagunes, García y Barragán (2008) que consiste en: Análisis de frecuencia de cada reactivo, para conocer el tipo de distribución predominante, discriminación y direccionalidad de los reactivos, confiabilidad inicial, análisis de correlación, análisis factorial y confiabilidad total y por factores. Resultados: Los resultados muestran que de los 55 reactivos iniciales, posterior al análisis estadístico, finalmente se obtuvieron 15 reactivos divididos en 2 factores (Dirigido a la emoción evitativo y Dirigido a la emoción emocional negativo) y 3 indicadores (Dirigido al problema directo, Dirigido al problema revaluación cognitiva positiva y Revaluación cognitiva negativa). Conclusión: El IEECPI, es un instrumento válido, confiable y culturalmente relevante para evaluar estrategias de enfrentamiento en CPI de pacientes oncológicos y detectar necesidades con necesidad de intervención.

Palabras clave: Cuidadores; paciente oncológico; cáncer; estrategias de enfrentamiento.

1 División de Investigación y Posgrado, Residencia en Medicina Conductual, Facultad de Estudios Superiores Iztacala. Universidad Nacional Autónoma de México, México.

E-mail: fatima.espinoza.14@gmail.com

2 Facultad de Psicología. Universidad Nacional Autónoma de México, México.

E-mail: lisabel@unam.mx

3 Facultad de Psicología. Universidad Nacional Autónoma de México, México.

E-mail: eduardorojanova@gmail.com

4 División de Investigación y Posgrado, Residencia en Medicina Conductual, Facultad de Estudios Superiores Iztacala. Universidad Nacional Autónoma de México, México.

E-mail: crisbravo@comunidad.unam.mx

5 Hospital Juárez de México, México.

E-mail: armando7771@hotmail.com

* Dirección de correspondencia: Fatima Sagrario Espinoza Salgado. División de Investigación y Posgrado. Facultad de estudios superiores Iztacala. UNAM. Av. De los Barrios \#1 Fracc. Jardines de los Reyes. Tlalnepantla, México CP54090. E-mail: fatima.espinoza.14@gmail.com 


\title{
[en] Scale for coping strategies for informal caregivers of patients with cancer
}

\begin{abstract}
Objective: Analize the psychometric properties of a new instrument valid and reliable instrument, which assesses the coping strategies in informal primary caregivers (IPC) of patients with cancer. The Instrument of Coping Strategies in Primary Informal Caregivers (IEECPI), consists in 2 factors (EmotionFocused) and 3 indicators (Problem-Focused). Method: Has been evaluated 20 IPC cancer patients with a questionnaire of open questions, to get information about the conceptualization of the theoretical dimensions of the coping strategies, been getting 216 indicators, that were grouped in two categories: Focused emotion and Focused problem, of which 55 reagents that were applied to 255 IPC were generated. Once the data proposed by Nunnally and Bernstein (1994) y el de Reyes-Lagunes, García y Barragán (2008) procedure is followed: frequency analysis of each item, to determine the predominant type of distribution, discrimination and directionality item, starting reliability, correlation analysis, factor analysis and full and reliability factors. Results: The results show that the 55 items at the begging, after statistical analysis, finally 15 items divided into two factors (Appraisal Focused strategies to the avoidant emotion and focused to the negative emotion emotional) and 3 indicators (Focused- problem were obtained focused problem positive and negative cognitive, appraisal cognitive). Conclusion: The IEECPI, it is a valid, reliable and culturally relevant to evaluate coping strategies in ICP of cancer patients and identify needs of intervention instrument. Keywords: Caregivers; cancer patients; cancer; coping strategies.
\end{abstract}

Sumario. 1. Introducción 2. Método 3. Discusión 4. Referencias bibliográficas.

Cómo citar: Espinoza-Salgado FS, Reyes-Lagunes I; Velasco-Rojano E, Bravo-González MC, Ramírez Ramírez A. Escala de estrategias de enfrentamiento para cuidadores primarios informales de pacientes con cáncer. Psicooncología 2017;14: 121-136. DOI: 10.5209/PSIC.55816

\section{Introducción}

A lo largo de la vida el ser humano atraviesa por diversas situaciones; algunas de ellas son estresantes y las personas pueden responder de diversas formas, por ejemplo: algunas se desesperan cuando las cosas no salen como las planearon; otras son capaces de afrontar retos y problemas de forma ecuánime, muchas otras pueden parecer estar tristes, pero dicen que están aprendiendo de esta situación. Uno de los sucesos que puede atravesar una persona a lo largo de la vida, puede ser recibir un diagnóstico de cáncer ${ }^{(1)}$, sin embargo, no sólo resulta estresante para el paciente sino también para la familia que lo rodea y apoya en este momento, debido a que esta situación conllevará a un cambio importante en su estilo de vida; este cambio se dará fundamentalmente por la limitación de capacidades que el paciente tendrá y como consecuencia necesitará de cuidados. Para ello, de forma circunstancial, uno de los miembros de la familia, regularmente asume la mayor parte de responsabilidad del cuidado del paciente, de forma implícita e inesperada y, es así como se convierte en el Cuidador Primario Informal (CPI) quien a lo largo del proceso de enfermedad del paciente, invertirá gran parte de su día en actividades como: Preparar alimentos, acudir a las citas médicas con el enfermo, proveer apoyo emocional, ayudar a los pacientes a tomar decisiones o tomarlas ellos, ayudar o realizar actividades de autocuidado del paciente ${ }^{(2-5)}$.

El cuidado de un familiar puede ser prolongado, y conlleva a que el cuidador realice cambios dentro de su vida social, laboral, familiar, todo ello en función de las tareas de cuidado del paciente; sin embargo, es importante considerar que el tipo de tareas de cuidado dependerán de la etapa de la enfermedad en la que se 
encuentre el paciente ${ }^{(6,7)}$. Dichos cambios y demandas que el cuidador adquiere al asumir este rol, generan coste de respuesta importantes, pareciendo tareas interminables que en ocasiones se perciben como desbordantes siendo así una notable fuente de estrés, esto pone al CPI en una situación de vulnerabilidad ante enfermedades físicas y/o problemas emocionales ${ }^{(6,8)}$.

El cuidado de un paciente con cáncer, para la mayoría de los CPI, suele ser una situación nueva, por lo que no cuentan con un aprendizaje previo para manejar esta situación, lo cual aumenta la percepción de estrés, una de las razones está relacionada con las estrategias de enfrentamiento que el CPI pone en marcha para el evento estresante y, al ser una situación no experimentada con anterioridad, el CPI hace uso de estrategias que en una situación diferente le fueron funcionales y que, en este momento, considera que sucederá lo mismo, sin embargo no necesariamente ocurre asi $i^{(2,6,7)}$.

Estas estrategias, fueron descritas por Lazarus y Folkman y Lazarus, $\mathrm{B}^{(9,10)}$ con el término original en inglés "coping", que tiene por significado: Lidiar con los problemas para tener éxito tratando e intentando superarlos y resolverlos, esforzándose y encontrando recursos para ellos. Sin embargo, al realizar la traducción del término "Coping", al español, parece ser que la palabra enfrentamiento representa mejor el significado del concepto, porque significa: Tener enfrente, luchar y/o superar los problemas; por ello a lo largo de esta investigación se utilizará el concepto de enfrentamiento ${ }^{(10,12)}$.

Las estrategias de enfrentamiento son todas aquellas alternativas o técnicas que el individuo utiliza para reducir el estrés. Una persona al estar sometida a estrés, selecciona y utiliza las estrategias dependiendo de factores tanto internos como externos: los primeros son las características del individuo como salud, energía, creencias, valores, déficits psicológicos y habilidades para resolver problemas; mientras que, los segundos son la amenaza percibida, el tipo de situación y las redes de apoyo social ${ }^{(9,13)}$ y es probable que cada individuo utilice las estrategias que en situaciones similares anteriores le han sido funcionales, pero si éstas no tienen éxito seguramente utilizará otra estrategia, lo cual sería una situación de aprendizaje por ensayo y error ${ }^{(9,10,13)}$. De acuerdo con Lazarus y Folkman ${ }^{(14)}$, el enfrentamiento hace referencia a "Los esfuerzos cognitivos y conductuales constantemente cambiantes que se desarrollan para manejar las demandas específicas externas y/o internas que son evaluadas como excedentes o desbordantes de los recursos del individuo, cualquier intento para dominar una situación nueva que puede ser potencialmente amenazante, frustrante, cambiante o desafiante" p. 141.

Estas estrategias se dividen en dos tipos: las que se dirigen al problema y las dirigidas a la emoción.

Las estrategias dirigidas al problema son las que tienen por objetivo la búsqueda de soluciones alternativas, a la consideración de tales alternativas con base a su costo-beneficio y a su elección de aplicación. La función de estas estrategias es manejar la fuente del estrés y predominan cuando la persona considera que puede hacerse algo eficaz. Existen dos tipos de estrategias dirigidos al problema, el Enfrentamiento directo, donde la persona realiza una conducta orientada a resolver el problema, se detiene poco a reflexionar que emoción percibe; actúa de forma inmediata, accede a sus recursos para abordar el problema. Por otro lado, la Reevaluación Cognitiva consiste en que el individuo en primer lugar realiza una valoración interna del problema, considerando cambios motivacionales el individuo da una respuesta dirigida a resolver la fuente estrés ${ }^{(9,10,13-14)}$. 
Las estrategias de enfrentamiento dirigidas a la emoción, son procesos cognitivos encargados de disminuir y/o regular la respuesta emocional, se utilizan en prácticamente cualquier situación estresante que el sujeto considere que la situación es perturbable y que puede hacer muy poco o nada para resolverlo. Dentro de las estrategias centradas en la emoción existen tres tipos, en primer lugar, se encuentran las estrategias de tipo Evasivo: Se centra en no pensar en aquello que preocupa al sujeto o realizar cualquier actividad que evite enfrentarse al problema. Se considera una mala estrategia de enfrentamiento, poco funcional porque, aunque las preocupaciones pueden parecer menos graves, los problemas no desaparecen, siguen presentes; La estrategia de tipo Emocional Negativo: Expresa una emoción pe. enojo, angustia, tristeza y no ayuda a resolver el problema; de igual forma es una estrategia poco efectiva, que tiene por consecuencia que la situación estresante continúe afectando física y emocionalmente al individuo; Por último, se encuentra la estrategia de tipo Revalorativa, donde el individuo da un sentido positivo al problema, trata de aprender de la situación o de alguna manera mejora su percepción. Este proceso cambia el significado personal de lo ocurrido evaluando de manera más sensata, serena y menos amenazadora; ésta debe de ser creíble de lo contrario se asume como una negación ${ }^{(9,10)}$.

Como se mencionó anteriormente, la percepción de estrés en los CPI, en ocasiones, se encuentra relacionada con las estrategias que utilizan para enfrentarlo por lo que es importante evaluar estas estrategias ${ }^{(2)}$. De acuerdo con la literatura, en anteriores estudios el instrumento más utilizado ha sido Ways of Coping Questionnaire (WOQ), que ha sido validado en diferentes poblaciones, por ejemplo: en CPI de pacientes con demencia; en CPI de pacientes en cuidados paliativos; en pacientes con enfermedades crónicas degenerativas, ya sea adaptada para la investigación o se utiliza una adaptación previa $^{(15,16)}$. En otras investigaciones, se utilizan escalas existentes y que se adaptan para poblaciones de Cuidadores Primarios Informales ${ }^{(2-4,17)}$. Estas evidencias muestran que como tal no existe una escala de Estrategias de Enfrentamiento, diseñada para Cuidadores Primarios Informales de habla hispana y culturalmente relevante ${ }^{(18)}$; esto es de importante consideración, porque los problemas o situaciones estresantes a las que se enfrentan los CPI, tienden a ser muy específicas, por ejemplo: El aumento de actividades diarias, el abandono del trabajo remunerado, la falta de dinero, el peligro inminente de muerte, desatender algunas otras actividades, etc. ${ }^{(16)}$. Con base en lo anterior, resulta indispensable contar con instrumentos que permitan identificar las estrategias de enfrentamiento de los Cuidadores Primarios Informales, por lo que, es el objetivo del presente estudio generar un instrumento que permita identificar las estrategias de enfrentamiento en los Cuidadores Primarios Informales de pacientes con cáncer en una población mexicana de manera confiable y válida.

\section{Método}

La presente investigación se divide en dos fases:

1. La obtención de indicadores, para recabar información sobre cómo la población meta conceptualiza las dimensiones teóricas de las estrategias de enfrentamiento.

2. La aplicación del instrumento basado en las preguntas de la fase anterior para obtener los items válidos y confiables que formarán la escala final. 


\section{Fase 1. Obtención de indicadores}

\section{Participantes}

Se evaluaron a 20 personas, mayores de edad, quienes informaron ser cuidadores de pacientes oncológicos de un hospital de tercer nivel. 15 participantes mujeres ( $75 \%$ de la muestra) y 5 hombres ( $25 \%$ de la muestra); con un promedio de edad de 45,8 años ( $\mathrm{SD}=12,36$, rango de edad de 26 a 67 años).

\section{Instrumento}

Se utilizó el cuestionario de 11 preguntas abiertas diseñado para el estudio, las preguntas fueron creadas por los autores de este estudio y el criterio para realizarlas fue de acuerdo a los niveles de respuesta que señalan Lazarus y Folkman; y Lazarus y Lazarus $^{(9,10,13)}$ (cognitivo, conductual y emocional), para las estrategias de enfrentamiento; algunas de estas preguntas eran: ¿Qué hace usted ante el cuidado de su paciente?, ¿Qué cosas piensa sobre cuidar a su paciente?, ¿Qué siente sobre cuidar a su paciente?.

\section{Procedimiento}

El cuestionario de preguntas abiertas se aplicó a 20 participantes en la sala de espera del servicio de Oncología, se obtuvo su consentimiento, respuestas y finalmente se agradeció su participación.

\section{Resultados}

En total se obtuvieron 216 indicadores, para lo cual fue necesario construir un plan de prueba ${ }^{(18)}$, donde de acuerdo a la teoría Lazarus y Folkman, y Lazarus, y Lazarus $^{(9,10)}$, se describen las categorías y subcategorías de esta teoría de acuerdo a los 3 niveles de respuesta (conductual, cognitivo y emocional) y se otorga un porcentaje a cada categoría, para determinar el número de ítems que corresponderá a ese nivel hasta formar un $100 \%$, que describe por completo el constructo, en este caso estrategias de enfrentamiento. De acuerdo al número de indicadores obtenidos en cada categoría, se agruparon los porcentajes de acuerdo a la teoría antes mencionadas ${ }^{(9,10)}$ y los tres niveles de respuesta, para saber qué subcategorías tenían el mayor número de indicadores, con los que se formularían los reactivos del instrumento, encontrando los resultados que se muestran en la Tabla 1.

Tabla 1. Porcentaje de indicadores obtenidos de acuerdo a la subcategoría.

\begin{tabular}{lc}
\hline \multicolumn{1}{c}{ Subcategoría } & $\% /$ Peso \\
\hline Dirigido al problema, subcategoría Directo: & $27,3 \%$ \\
Dirigido a la emoción, subcategoría Revalorativo: & $25,0 \%$ \\
Dirigido al problema, subcategoría Revalorativo Cognitivo: & $19,9 \%$ \\
Dirigido a la emoción, subcategoría Negativo Emocional: & $13,4 \%$ \\
Dirigido a la emoción, subcategoría Emergente: & $7,4 \%$ \\
Dirigido al problema, subcategoría Emergente: & $6,4 \%$ \\
Dirigido a la emoción, subcategoría Evitativo: &, $4 \%$ \\
\hline
\end{tabular}


Después de ello, se generaron 55 reactivos, tomando como criterio que cada categoría debería tener de 4 a 5 reactivos, en primer lugar, porque no en todas las subcategorías se obtuvo el mismo número de indicadores y en segundo lugar porque es el número adecuado para que en su posterior análisis obtener factores significativos de acuerdo con el método de Reyes-Lagunes, García y Barragán ${ }^{(18)}$, divididos dentro de las subcategorías en 3 dimensiones (cognitivo, emocional y conductual), que de acuerdo a las teorías ${ }^{(9,10)}$, sé describen, como se muestra en la Tabla 2 en el plan de prueba.

Tabla 2. Plan de prueba con los porcentajes de los indicadores obtenidos.

\begin{tabular}{|c|c|c|c|c|}
\hline Categorías & Subcategorías & $\begin{array}{l}\text { COGNOSCITIVA } \\
\text { Variar el nivel de } \\
\text { aspiraciones. }\end{array}$ & $\begin{array}{l}\text { EMOCIONAL } \\
\text { Buscar canales } \\
\text { de gratificación }\end{array}$ & $\begin{array}{c}\text { CONDUCTUAL } \\
\text { Modificar el } \\
\text { entorno }\end{array}$ \\
\hline \multirow{3}{*}{$\begin{array}{l}\text { Dirigido } \\
\text { a la emoción }\end{array}$} & Evasivo & $9 \%$ & $\begin{array}{l}\text { No descrito por } \\
\text { la teoría }\end{array}$ & $9 \%$ \\
\hline & $\begin{array}{l}\text { Emocional } \\
\text { Negativo }\end{array}$ & $9 \%$ & $9 \%$ & $9 \%$ \\
\hline & Revalorativo & $9 \%$ & $10 \%$ & $\begin{array}{l}\text { No descrito por } \\
\text { la teoría }\end{array}$ \\
\hline \multirow{2}{*}{$\begin{array}{l}\text { Dirigido al } \\
\text { problema }\end{array}$} & $\begin{array}{c}\text { Afrontamiento } \\
\text { directo }\end{array}$ & $\begin{array}{c}\text { No descrito por la } \\
\text { teoría }\end{array}$ & $\begin{array}{l}\text { No descrito por } \\
\text { la teoría }\end{array}$ & $9 \%$ \\
\hline & $\begin{array}{c}\text { Revaluación } \\
\text { cognitiva }\end{array}$ & $9 \%$ & $9 \%$ & $9 \%$ \\
\hline
\end{tabular}

\section{Fase 2. Validez y Confiabilidad del instrumento}

\section{Participantes}

255 Cuidadores Primarios Informales (CPI) de pacientes oncológicos de un Hospital de tercer nivel de la Ciudad de México, conformaron la muestra de estudio, $170(66,7 \%)$ eran mujeres, $85(33,3 \%)$ eran hombres. Con una media de edad de 45,28 años ( $\mathrm{SD}=12,53$, rango de edad de 19 a 73 años); en cuanto a la escolaridad un promedio de 7,2 años $(\mathrm{SD}=1,4)$, con un rango de escolaridad de 6 años (primaria incompleta) a 16 años (Posgrado). El 23,9\% solo cuenta con primaria, el 33,7\% tiene secundaria, el 11,4\% nivel técnico el 15,3\% cuenta con preparatoria, el 12,9\% reportó tener licenciatura y solamente el $2,7 \%$ con estudios de posgrado.

De acuerdo a las variables relacionadas con los pacientes, se encontró que 151 $(59,2 \%)$ participantes cuidaban a pacientes en fase de tratamiento, $39(15,3 \%)$ cuidaban a pacientes en fase pre-diagnóstica, $37(14,5 \%)$ en fase diagnóstica, 9 $(3,5 \%)$ estaban libres de enfermedad, $9(3,5 \%)$ en recaída, $9(3,5 \%)$ en fase paliativa y $1(0,4 \%)$ en fase terminal. En cuanto al parentesco que los CPI tenían con los pacientes se encontró que $105(41,2 \%)$ eran hijos del paciente, $36(14,1 \%)$ eran los esposos, $30(11,8 \%)$ eran esposas, $27(10,6 \%)$ eran hermanos, $35(13,7 \%)$ de ellos tenían otro parentesco (sobrino, tío, nuera, cuñado, suegra, etc.).

Respecto al tiempo de cuidado al día que dedican a su familiar, se obtuvo una media de 15,57 horas diarias ( $\mathrm{SD}=7,79$, rango de horas 6 a 24 horas). En lo que se refiere a la duración de cuidado, se tomó como criterio de inclusión un mínimo 
de 2 meses de historia de cuidado, por lo tanto, se obtuvo una media de 6,6 meses $(\mathrm{SD}=2,2$ rango de meses 2 a 15 meses $)$.

\section{Instrumento}

Se aplicaron los 55 reactivos construidos, producto de la Fase 1 de desarrollo de la escala, con opciones de respuesta de tipo Likert que van de Totalmente en desacuerdo (0) a Totalmente de Acuerdo (3).

\section{Procedimiento}

Se construyeron los 55 reactivos iniciales y, para su aplicación, se contactó a los participantes en un hospital de tercer nivel de la Ciudad de México, en el área de hospitalización y en la sala de espera de consulta externa; se aplicó el instrumento después de obtener su consentimiento y brindar las instrucciones, al final se agradeció su participación.

Una vez obtenidos los datos se siguió el procedimiento propuesto por Nunnally y Bernstein $^{(19)}$ y el de Reyes-Lagunes, García y Barragán ${ }^{(18)}$ que consiste en:

- Análisis de frecuencia de cada reactivo para conocer, si las opciones de respuesta fueron atractivas para todos los participantes y conocer el tipo de distribución predominante: normal o sesgada.

- Discriminación y direccionalidad de los items.

- Confiabilidad inicial.

- Análisis de correlación para decidir el tipo de análisis factorial.

- Análisis Factorial.

- Confiabilidad total y por factores.

- Obtención de percentiles.

Con los datos obtenidos de los reactivos válidos y confiables se obtuvieron los percentiles, de cada factor y sus respectivos puntos de corte.

\section{Resultados}

Con respecto al análisis de frecuencias de cada reactivo, se obtuvo que los 55 items presentaron frecuencia en todas y cada una de sus opciones de respuesta. A partir de conocer el tipo de distribución predominante normal o sesgada, se tomó la decisión de eliminar 8 reactivos por tener distribuciones normales, para seguir trabajando con los de distribución sesgada, por ser predominantes. En cuanto a la discriminación y direccionalidad de reactivos, se eliminaron 4 reactivos y 16 necesitaron recodificación. Por último, se realizó un análisis de correlación entre todos los items, para evaluar que tengan una relación significativa y esto determina que todos los ítems que correlacionen con el total están evaluando estrategias de enfrentamiento (las cosas que hace, piensa o siente) respecto al cuidado del paciente. Se observó que 14 de ellos no presentaban correlaciones altas, lo que se interpreta que no están evaluando estrategias de enfrentamiento, como puede verse en la Tabla 3.

De acuerdo con lo observado en el análisis de correlación entre los reactivos de predominancia alta, se decidió realizar un análisis factorial exploratorio de extracción de componentes principales con rotación ortogonal (Varimax Kaiser), para 21 reactivos restantes.

A partir del análisis factorial exploratorio, la escala queda integrada por dos factores y tres indicadores, con un porcentaje de varianza explicada del 58,17\%, con 15 elementos y un Alpha de Cronbach de 0,703 (ver Tabla 4). Los factores son: Estrategia 
de enfrentamiento dirigida a la emoción de tipo evitativo y Estrategia de enfrentamiento dirigida a la emoción de tipo emocional negativo. En lo que respecta a los indicadores son: Estrategia de enfrentamiento dirigida al problema de tipo directa, Estrategia de enfrentamiento dirigida al problema de tipo revaloración cognitiva positiva y Estrategias de enfrentamiento dirigida al problema de tipo revaloración cognitiva negativa.

Tabla 3. Proceso de discriminación de los reactivos de la escala de estrategias de enfrentamiento.

\begin{tabular}{|c|c|c|c|c|}
\hline Reactivo & Sesgo & $\begin{array}{c}\text { P en } \\
T\end{array}$ & $\begin{array}{c}\text { Correlación } \\
\text { reactivo- } \\
\text { total }\end{array}$ & Criterio \\
\hline $\begin{array}{l}\text { 1. Procuro que mi paciente no se entere de su } \\
\text { actual estado de salud. }\end{array}$ & 0,723 & ,000 &, $393 * *$ & \\
\hline $\begin{array}{l}\text { 2. Me pone muy contento (a) poder ser yo quien } \\
\text { lo cuide. }\end{array}$ & $-1,64$ &, 000 & $260 * *$ & Eliminado \\
\hline 3. Cuidar a mi paciente me genera preocupación &,- 398 &, 000 &, $356 * *$ & Eliminado \\
\hline $\begin{array}{l}\text { 4. El objetivo de cuidar a mi paciente es que me } \\
\text { agradezca lo que hago por él. }\end{array}$ & 1,724 &, 000 & ,336** & \\
\hline $\begin{array}{l}\text { 5. Siento que mi cuerpo tiembla, cuando cuido a } \\
\text { mi paciente. }\end{array}$ & ,935 & ,000 &, $469 * *$ & \\
\hline 6. Cuido con gusto a mi paciente. & $-2,74$ &, 015 &, $180 * *$ & Eliminado \\
\hline $\begin{array}{l}\text { 7. Estoy al pendiente de la alimentación de mi } \\
\text { paciente. }\end{array}$ & $-1,84$ &, 000 & $200 * *$ & \\
\hline 8. Cuidar a mi paciente es difícil para mí. & ,435 &, 000 &, $345 * *$ & Eliminado \\
\hline 9. Al cuidar a mi paciente me siento útil. & $-1,36$ &, 000 &, $346 * *$ & \\
\hline 10. Cuidar a mi paciente es mi obligación &,- 463 &, 000 &, $295 * *$ & Eliminado \\
\hline 11. Cuidar a mi paciente me provoca miedo. &, 745 & 000 &, $404 * *$ & \\
\hline $\begin{array}{l}\text { 12. He hecho algunas cosas para apoyar a mi } \\
\text { paciente. }\end{array}$ & $-1,48$ & , 127 &, 112 & Eliminado \\
\hline $\begin{array}{l}\text { 13. Me agrada que los demás reconozcan lo que } \\
\text { hago por mi familiar. }\end{array}$ & ,408 & ,000 & $241 * *$ & Eliminado \\
\hline $\begin{array}{l}\text { 14. Trato que los demás no se enteren de la } \\
\text { enfermedad de mi paciente. }\end{array}$ & ,713 & 000 &, $443 * *$ & \\
\hline $\begin{array}{l}\text { 15. Cuando cuido a mi paciente me siento muy } \\
\text { contento. }\end{array}$ & $-1,44$ & ,002 &, $182 * *$ & \\
\hline $\begin{array}{l}\text { 16. He intentado golpear a alguien, cuando realizan } \\
\text { comentarios sobre el cuidado de mi familiar. }\end{array}$ & 2,025 &, 000 & $341 * *$ & \\
\hline 17. Me desagrada cuidar a mí paciente. & 2,193 &, 001 &, $278 * *$ & Eliminado \\
\hline $\begin{array}{l}\text { 18. He hecho todo lo posible para que mi paciente } \\
\text { mejore. }\end{array}$ & $-2,41$ & ,419 &, 100 & Eliminado \\
\hline 19. Cuidar a mi paciente me provoca estrés & ,602 &, 000 &, $438 * *$ & \\
\hline $\begin{array}{l}\text { 20. Primero realizo otras actividades y dejo al } \\
\text { final el cuidado de mi paciente. }\end{array}$ & 1,587 &, 003 & $25 * *$ & Eliminado \\
\hline $\begin{array}{l}\text { 21. Le he dedicado tiempo al cuidado de mi } \\
\text { paciente. }\end{array}$ & $-1,49$ &, 000 &, $302 * *$ & \\
\hline 22. Con mis cuidados le demuestro mi amor. & $-1,94$ &, 004 &, $202 * *$ & Eliminado \\
\hline
\end{tabular}


23. Quisiera salir corriendo ante el cuidado de mi $1,191 \quad, 000 \quad$,392** paciente.

24. Sufro al cuidar a mi paciente.

,869, 000 ,531**

25. Pienso que el cuidado de mi paciente es mi $\quad-, 571,000 \quad, 374 * *$ responsabilidad

26. Temo perder a mi familiar.

27. He hecho algo por conseguir dinero.

$-1,20,000 \quad, 290 *$

Eliminado

28. Me da alegría ver que lo que hago por mi

$-, 571,000$

,303**

$-2,54,000 \quad, 320 * *$ paciente, tiene resultados.

29. Hago cosas como: Tomar alcohol, drogarme, para evitar pensar en el cuidado de mi paciente.

30. He brindado muestras de afecto a mi paciente (Consentirlo, mimarlo, apapacharlo etc.), para que se sienta mejor.

31. Cuidar a mi paciente es pesado.

$3,769,000 \quad, 267 * * \quad$ Eliminado

$-2,00,000 \quad, 328 * *$

32. Si descuido a mi paciente, me siento una persona desobligada.

33. Estoy al pendiente de los medicamentos que debe de tomar y le deben aplicar a mi paciente.

$\begin{array}{cccc}, 371 & , 000 & , 327 * * & \text { Eliminado } \\ -, 512,000 & , 339 * * & \\ -1,57 & , 001 & , 195 * * & \text { Eliminado } \\ & & & \\ , 522 & , 000 & , 399 * * & \\ , 045 & , 010 & , 177 * * & \text { Eliminado } \\ -1,89 & , 000 & , 290 * * & \text { Eliminado } \\ 2,11 & , 014 & , 177 * * & \end{array}$

34. Cuidar a mi paciente me genera angustia.

35. Pienso cosas diferentes al cuidado de mi paciente.

36. Cuido a mi paciente, para que se sienta apoyado.

37. He golpeado objetos (pared, puertas) ante el cuidado de mi paciente.

38. He apoyado a mi paciente en todo lo posible.

39. El objetivo de mi cuidado es que mi paciente mejore.

40. Pienso que es mi deber cuidar a mi familiar.

41. Lloro cuando cuido a mi paciente.

, $177 *$

$-2,54,000 \quad, 261 * *$

Eliminado

$-2,50,262,201 * * \quad$ Eliminado

42. Siento tranquilidad al cuidar a mi paciente.

43. La desventaja de cuidar a mi familiar es no tener dinero.

44. Cuidar a mi paciente me genera insatisfacción.

45. Evito realizar actividades relacionadas con el cuidado de mi paciente.

$-, 941,000 \quad, 403 * *$

$, 781,000 \quad, 378 * *$

$-1,29,006 \quad, 201 * * \quad$ Eliminado

$-, 057,000 \quad, 340 * * \quad$ Eliminado

$1,662,040 \quad, 256^{* *} \quad$ Eliminado

$, 897,000 \quad, 254 * * \quad$ Eliminado

46. Los cuidados que ha dado a su paciente, han $\quad-1,67,647 \quad, 107 \quad$ Eliminado sido útiles.

47. Evito pensar en el cuidado de mi paciente. $\quad 1,175,000 \quad, 354^{* *}$

48. Cuidar a mi paciente me provoca tristeza. $\quad$, 691 ,000 ,564**

49. Cuidar a mi paciente me provoca cansancio. $\quad 120 \quad, 000 \quad, 362 * *$

50. Me siento bien cuando cuido a mi paciente. $\quad-1,62,003 \quad, 233^{* *} \quad$ Eliminado

51. Si descuido a mi paciente, me siento irresponsable

$-, 677,000 \quad, 325^{* *}$

\footnotetext{
** Correlaciones significativas al ,001 y sólo se eliminan las correlaciones $>, 300$, que de acuerdo con Pearson se consideran muy débiles.
} 
Tabla. 4. Análisis factorial de extracción de componentes principales con rotación ortogonal.

\begin{tabular}{|c|c|c|c|c|c|}
\hline Reactivo & Factor 1 & Factor 2 & Factor 3 & Factor 4 & Factor 5 \\
\hline Cuidar a mi paciente me genera angustia & ,747 &, 030 & ,086 &, 080 &,- 104 \\
\hline Cuidar a mi paciente me provoca miedo & ,700 & ,048 &,- 053 & ,094 & ,048 \\
\hline $\begin{array}{l}\text { Siento que mi cuerpo tiembla, cuando } \\
\text { cuido a mi paciente }\end{array}$ & ,697 & ,258 &, 028 &,- 085 & ,068 \\
\hline Cuidar a mi paciente me genera tristeza & ,690 & ,332 & 074 &,- 014 &,- 026 \\
\hline $\begin{array}{l}\text { Trato que los demás no se enteren de la } \\
\text { enfermedad de mi paciente }\end{array}$ &, 110 & ,643 & ,029 & ,056 & , 135 \\
\hline $\begin{array}{l}\text { Procuro que mi paciente no se entere de } \\
\text { su estado actual de salud }\end{array}$ &, 145 & ,631 &,- 280 & , 126 &,- 010 \\
\hline $\begin{array}{l}\text { He intentado golpear a alguien cuando } \\
\text { realiza comentarios sobre el cuidado de } \\
\text { mi familiar }\end{array}$ &, 028 & ,614 &,- 005 & ,081 & ,002 \\
\hline $\begin{array}{l}\text { Quisiera salir corriendo ante el cuidado } \\
\text { de i paciente }\end{array}$ &, 139 &, 575 & 087 & 029 &,- 076 \\
\hline Lloro cuando cuido a mi paciente & ,276 &, 511 & ,232 &,- 105 &,- 131 \\
\hline $\begin{array}{l}\text { Si descuido a mi paciente, me siento } \\
\text { irresponsable }\end{array}$ & ,073 &,- 004 & ,830 &, 068 &, 047 \\
\hline $\begin{array}{l}\text { Si descuido a mi paciente, me siento una } \\
\text { persona desobligada }\end{array}$ & 024 &, 051 &, 826 &, 176 &, 144 \\
\hline $\begin{array}{l}\text { Pienso que es mi deber cuidar a mi } \\
\text { familiar }\end{array}$ &, 028 & 073 &, 114 & ,854 & 047 \\
\hline $\begin{array}{l}\text { Pienso que el cuidado de mi paciente es } \\
\text { mi responsabilidad }\end{array}$ & ,315 & ,505 & ,485 & ,847 &,- 303 \\
\hline $\begin{array}{l}\text { He brindado muestras de afecto a } \\
\text { mi paciente (Consentirlo, mimarlo, } \\
\text { apapacharlo, etc.), para que se sienta } \\
\text { mejor. }\end{array}$ &,- 073 & ,091 & ,132 & ,827 & ,827 \\
\hline $\begin{array}{l}\text { Le he dedicado tiempo al cuidado de mi } \\
\text { paciente }\end{array}$ & 057 &,- 109 & ,041 & ,084 & ,823 \\
\hline$\%$ de varianza explicada & 14,38 & 13,33 & 10,46 & 10,33 & 9,65 \\
\hline Alpha de Cronbach & ,712 & ,596 & 674 & ,687 &, 580 \\
\hline $\begin{array}{l}\text { Alpha de Cronbach total de la escala } 11 \\
\text { elementos }\end{array}$ & ,707 & & & & \\
\hline
\end{tabular}

Para verificar que la matriz de correlaciones sobre la cual se basa el análisis factorial fuera adecuada, se realizaron dos análisis: la medida de adecuación muestral Kaiser, Meyer, Olkin $(\mathrm{KMO}=0,690)$ y la prueba de esfericidad de Barttlet $\left(\mathrm{X}^{2}=\right.$ $675,644, \mathrm{p}<0,00001)$ a partir de las cuales, se llegó a la conclusión de que fueron adecuadas. 
Para evaluar las relaciones entre los componentes de la escala, se llevaron a cabo correlaciones de Pearson entre los factores, las cuales se aprecian significativas (ver Tabla 5).

Tabla 5. Relación entre factores (Producto-momento de Pearson).

\begin{tabular}{|c|c|c|c|c|c|}
\hline Factores & $\begin{array}{l}\text { Dirigida a } \\
\text { la emoción } \\
\text { Evitativo }\end{array}$ & $\begin{array}{c}\text { Dirigida a } \\
\text { la emoción: } \\
\text { Emocional } \\
\text { negativo }\end{array}$ & $\begin{array}{l}\text { Dirigida } \\
\quad \text { al } \\
\text { problema } \\
\text { Directo }\end{array}$ & $\begin{array}{c}\text { Dirigida al } \\
\text { problema } \\
\text { Revaluación } \\
\text { cognitiva } \\
\text { positiva }\end{array}$ & $\begin{array}{c}\text { Dirigida al } \\
\text { problema } \\
\text { Revaluación } \\
\text { cognitiva } \\
\text { negativa }\end{array}$ \\
\hline $\begin{array}{l}\text { Dirigida a la emoción } \\
\text { Evitativo }\end{array}$ & 1 & & & & \\
\hline $\begin{array}{l}\text { Dirigida a la emoción: } \\
\text { Emocional negativo }\end{array}$ & ,431** & 1 & & & \\
\hline $\begin{array}{l}\text { Dirigida al problema } \\
\text { Directo }\end{array}$ &, 058 & ,113 & 1 & & \\
\hline $\begin{array}{l}\text { Dirigida al problema } \\
\text { Revaluación cognitiva } \\
\text { positiva }\end{array}$ &, $164 * *$ & ,094 &, $239 * *$ & 1 & \\
\hline $\begin{array}{l}\text { Dirigida al problema } \\
\text { Revaluación cognitiva } \\
\text { negativa }\end{array}$ &,- 027 &,- 018 &, $186^{* * *}$ &, 119 & 1 \\
\hline
\end{tabular}

** La correlación es significativa al nivel 0,01 .

Por último se realizó la obtención de percentiles, por cada factor e indicador, para su calificación y diagnóstico.

\section{Discusión}

Del procedimiento de preguntas abiertas se obtuvieron 55 reactivos, de los que finalmente se obtuvieron 15 reactivos divididos en 2 factores (Dirigido a la Emoción Evitativo y Dirigido a la Emoción Emocional negativo) y 3 indicadores (Dirigido al problema directo, Dirigido al problema revaluación cognitiva positiva y revaluación cognitiva negativa). Cabe mencionar, que los factores emocionales no presentan correlaciones significativas con los indicadores dirigidos al problema, pareciendo ser estrategias diferentes; sin embargo, entre las subcategorías si existen correlaciones significativas. Estos resultados pueden atribuirse a que el enfrentamiento es un proceso multifactorial que abarca situaciones y características personales. Existen distintos eventos estresantes, así como diferentes formas de enfrentarlos y el cuidado de un paciente es una situación estresante que para la mayoría de los cuidadores resulta novedosa y que surgió de manera circunstancial ${ }^{(16)}$. Es por ello que en esta validación la categoría Dirigido al Problema, únicamente, arroja indicadores válidos y confiables, porque la mayoría de los CPI a pesar del malestar emocional generado 
por el estrés deben y realizan actividades, porque de ello depende la vida de su paciente y para llevarlas a cabo realizan revaluaciones constantes, pero el hecho de que realicen actividades que se dirigen al problema, no quiere decir que el cuidador no tenga estrategias dirigidas a la emoción, particularmente porque el cuidado por sí mismo es una situación estresante que genera malestar emocional y es hacia la disminución de éste que se dirigen dichas estrategias ${ }^{(1,3,6,17)}$. Por ello es que las correlaciones entre factores e indicadores Estrategias Dirigidas a la Emoción y Estrategias Dirigidas al Problema no resultaron significativas.

Es también de suma importancia mencionar, que la muestra estuvo compuesta por $33,3 \%$ de hombres y $67,3 \%$ de mujeres, siendo estas últimas las de mayor proporción y de acuerdo a un estudio realizado por Houtman ${ }^{(20)}$, donde encontró que las mujeres reportan más eventos estresantes en su vida, tal vez esto se debe a que culturalmente ellas han aprendido a expresar de forma más clara las emociones y podría confundirse con las estrategias de enfrentamiento dirigidas a la emoción. A su vez, podría atribuirse que las respuestas que dieron a los instrumentos provocaron mayor variabilidad en dichos ítems, dando como resultado factores fiables y confiables en Estrategias de Enfrentamiento Dirigidas a la Emoción. El que la cantidad de hombres haya sido menor, se reflejó en que los ítems dirigidos al problema, únicamente se obtuvieron como indicadores, más no como factores, al respecto Zeidner ${ }^{(21)}$ reporta que los hombres se perciben con mayor capacidad de hacer cosas para enfrentar un problema (estrategias dirigidas al problema) y no las estrategias contrarias porque no son socialmente aceptadas para su género.

Por otro lado, debe de tomarse en cuenta, que la teoría seleccionada para realizar este instrumento fue realizada y estudiada en un país con características diferentes a México y la gente en diferentes culturas ve los problemas de diferente forma. Por ello resulta importante plantear el estudio del enfrentamiento y control de los problemas de acuerdo con los ojos de la propia cultura ${ }^{(11,18)}$. Si bien es válido comparar y tomar en cuenta los resultados obtenidos con otras teorías aplicadas a lo largo del mundo, es importante tener en cuenta que los conceptos se perciben diferentes en las distintas culturas y el concepto enfrentamiento tiene su origen en una cultura distinta a la mexicana $^{(11)}$.

Por ejemplo, en México se ha descrito que los estrategias de enfrentamiento en su mayoría están dirigidas a la emoción, después a la autorregulación y por último al problema; estas características se ven afectadas por el nivel de escolaridad que predomina en nuestro país ${ }^{(12)}$ y la muestra con la que se construyó esta escala no es la excepción, porque el $57,6 \%$ de ésta contaba con nivel básico (primaria y/o secundaria), lo cual es un factor que predispone a presentar un pensamiento de tipo concreto, que los lleva a solucionar los problemas con estrategias de enfrentamiento dirigidas al malestar inmediato, en este caso a la emoción y lo perciben de forma separada a las dirigidas al problema. En lo que respecta a la fase de la enfermedad en la que se encontraban los pacientes de los cuidadores, la muestra no fue homogénea, debido a que el mayor porcentaje $(59,3 \%)$ de los participantes se encontraba al cuidado de un paciente en fase de tratamiento y de acuerdo con Chacín y Chacín ${ }^{(22)}$ los cuidadores en esta fase dirigen sus estrategias hacia la solución del problema. Los cuidadores que se esperaría que tuvieran estrategias de enfrentamiento dirigidas a la emoción son aquellos que están al cuidado de un paciente en fase diagnóstica, que son los menos $(3,7 \%)$, y probablemente esos resultados son los que generaron la variabilidad necesaria para la fiabilidad de los ítems obtenidos. 
Por último, es importante mencionar que los factores obtenidos: Dirigido a la Emoción de tipo evitativo y Dirigido a la emoción de tipo emocional negativo, dentro de esta escala serán útiles para evaluar a los CPI, porque si muestran mayor puntaje en estos factores quiere decir que muestran una estrategia de enfrentamiento poco funcional, lo cual los hace candidatos a una intervención psicológica para mejorar estas estrategias y ser más funcionales ante el cuidado de los pacientes.

Como conclusión, se obtuvo un instrumento que evalúa estrategias de enfrentamiento, tanto dirigidas a la emoción (Evitativo, Emocional Negativo) y proporciona indicadores sobre las estrategias de enfrentamiento dirigidas al problema (Directo, Revalorativo) de manera confiable y válida, construido eficazmente para Cuidadores Primarios Informales de pacientes con cáncer mexicanos (Anexo 1), lo que garantiza la relevancia cultural para la población meta, y que puede ser útil en futuras investigaciones así como en el ámbito de la psicología clínica, para brindar programas de intervención a esta población vulnerable.

\section{Referencias bibliográficas}

1. Moral R, Martínez, R. Reacción ante el diagnóstico de cáncer de un hijo: Estrés y afrontamiento. Psicol Salud 2009;19: 189-196.

2. Gaugler J, Eppinger A, King J, Sandberd T, Regine W. Coping and this effects on cancer caregiving. Support Care Cancer 2013; 22:385-395 Doi: 10,1007/s00520-012-1525-5

3. Bonnaud A, Hardouin J, Leger J, Drave F, Sebile V, Quality of life and coping of women treated for breast cancer and their caregiver. What the interactions? J Clin Psychol Med Settings 2012; 19: 320-328. Doi: 10.1007/S 10880-012-9300-9

4. Han Y, Hu D, Liu Y, Caihong, L, Luos Z, Zhao J, et al. Coping styles and socal support among depressed Chinese family caregivers of patients with esophageal cancer. EJON 2014;18:571-7. Doi: 10.1016/ j.ejon-2014 07002

5. Montero X. Valoración integral y factores de cambio en la carga de cuidadores primarios informales de niños con cáncer. México: Libros UNAM, 2013. Disponible en: http://132.248.204.112:8080/unamoa/handle/123456789/328.

6. Montero X, Jurado S, Méndez J. Variables que predicen la aparición de sobrecarga en cuidadores primarios informales de niños con cáncer. Psicooncología 2015; 12: 67-86. Doi: 10.5209/rev_PSIC.2015.v12.n1.48905.

7. Lawrence V, Murray J, Samsi K, Banerjee S. Attitudes and support needs of Black Caribbean, south Asia and White Britisth care of people with dementia in the UK. B J Psychiatry 2008; 193: 240-246 . Doi: 10.1192/bjp.bp.107.045187

8. McCorkle R, Given B. The emotional Responses of family caregivers: Living with cancer and helping families cope. En: Talle R, McCorkle R, Baile W, editors. Cancer caregiving in the United States. New York: Springer, 2012; p. 38-53.

9. Lazarus R, Folkman S. Stress, Appraisal and coping. New York: Springer, 1984.

10. Lazarus R, Lazarus, B. Pasion y razon: La comprensión de nuestras emociones. España: Paídos, 1994.

11. Góngora E. El enfrentamiento a los problemas y el papel del Control: Una visión etnopsicológica en un ecosistema con tradición. México: UNAM, 2000.

12. Ruíz B. Estilos de enfrentamiento, ansiedad y manejo del enojo en pacientes con hipertensión arterial esencial. México: UNAM, 2006. 
13. Lazarus R. Estrés y emoción. Manejo e implicaciones en nuestra salud. Bilbao: Descleé de Brouwer, 2000.

14. Lazarus R, Folkman S. Estrés y procesos cognitivos. México: Planeta Roca, 1991.

15. Rankin S. Influence of coping stlyes on social support seeking among cancer patient family caregivers. Estado Unidos: Walden University, 2011. [Acceso 1 de enero de 2017]. Disponible en: http://scholarworks.waldenu.edu/dissertations/908/

16. Tokem Y, Ozcelik H, Cicik A. Examination of the relationship between hopelessness levels and coping strategies among the family caregivers of patients wih cancer. Cancer Nurs 2015. 38: E28-34. Doi: 10.1097/NCC.0000000000000189.

17. Mosher C, Ott M, Hanna N, Jalal S, Champios, V. Coping with physical and psychological symptoms: A qualitative study of advance lung cancer patients and their family caregivers. Support Care Cancer 2015;23:2053-60. Doi: 10.1007/ a00520- 014-2566-8.

18. Reyes-Lagunes I, García y Barragán F. Procedimiento de Validación Psicométrica Culturalmente Relevante: Un ejemplo. En: Rivera S, Díaz-Loving R, Sánchez A, ReyesLagunes I, editores. La Psicología Social en México, México: Asociación Mexicana de Psicología Social, 2008. p. 625-36.

19. Nunnally J, Bernstein I. Psychometric Theory. New York: McGraw- Hill, 1994.

20. Houtman I. Personal coping resources and sex diferences. Pers Indvid Dif 1990;11:53-63

21. Zeidner M. Personal and contextual determinants of coping and anxiety in a evaluative situation: A perspective study. Pers Indiv Dif 1994;16:899-918.

22. Chacín F, Chacín J. Estrategias de Afrontamiento en padres de niños y adolescentes con cáncer. Rev Venez Oncol 2011;23:199-208. 


\section{ANEXO 1}

\section{INSTRUMENTO DE ESTRATEGIAS DE ENFRENTAMIENTO EN CUIDADORES PRIMARIOS INFORMALES}

A continuación encontrara una lista de situaciones que en general las personas piensan, sienten y/o hacen para enfrentar el cuidado de un paciente. Lea por favor cada una de las oraciones y marque con una cruz el número que corresponda a la categoría que mejor exprese lo que usted ha hecho a partir de que su familiar enfermó.

\section{Totalmente en Desacuerdo 0 En Desacuerdo 1 De Acuerdo 2 Totalmente de Acuerdo 3}

\begin{tabular}{|c|c|c|c|c|c|}
\hline 1) & Procuro que mi paciente no se entere de su actual estado de salud. & 0 & 1 & 2 & 3 \\
\hline 2) & Siento que mi cuerpo tiembla, cuando cuido a mi paciente. & 0 & 1 & 2 & 3 \\
\hline 3) & Cuidar a mi paciente me provoca miedo. & 0 & 1 & 2 & 3 \\
\hline 4) & Trato que los demás no se enteren de la enfermedad de mi paciente. & 0 & 1 & 2 & 3 \\
\hline 5) & Le he dedicado tiempo al cuidado de mi paciente. & 0 & 1 & 2 & 3 \\
\hline 6) & $\begin{array}{l}\text { He intentado golpear a alguien, cuando realizan comentarios sobre el } \\
\text { cuidado de mi familia }\end{array}$ & 0 & 1 & 2 & 3 \\
\hline 7) & Quisiera salir corriendo ante el cuidado de mi paciente & 0 & 1 & 2 & 3 \\
\hline 8) & Pienso que el cuidado de mi paciente es mi responsabilidad & 0 & 1 & 2 & 3 \\
\hline 9) & $\begin{array}{l}\text { He brindado muestras de afecto a mi paciente (Consentirlo, mimarlo, } \\
\text { apapacharlo, etc.) }\end{array}$ & 0 & 1 & 2 & 3 \\
\hline 10) & Si descuido a mi paciente, me siento una persona desobligada & 0 & 1 & 2 & 3 \\
\hline 11) & Cuidar a mi paciente me genera angustia & 0 & 1 & 2 & 3 \\
\hline 12) & Pienso que es mi deber cuidar a mi familiar & 0 & 1 & 2 & 3 \\
\hline 13) & Lloro cuando cuido a mi familiar & 0 & 1 & 2 & 3 \\
\hline 14) & Cuidar a mi paciente me genera tristeza & 0 & 1 & 2 & 3 \\
\hline 15) & Si descuido a mi paciente, me siento irresponsable & 0 & 1 & 2 & 3 \\
\hline
\end{tabular}

\section{CALIFICACIÓN}

\begin{tabular}{|l|c|c|c|}
\hline \multicolumn{1}{|c|}{$\begin{array}{c}\text { DIRIGIDO } \boldsymbol{A} \text { LA } \\
\text { EMOCIÓN }\end{array}$} & REACTIVOS & SUMA & PERCENTIL \\
\hline $\begin{array}{l}\text { Dirigida a la } \\
\text { emoción Evitativo }\end{array}$ & $6,7,13,14$ & & \\
\hline $\begin{array}{l}\text { Dirigida a la } \\
\text { emoción: Emocional } \\
\text { negativo }\end{array}$ & $2,3,11$ & & \\
\hline
\end{tabular}




\begin{tabular}{|l|c|l|l|}
\hline \multicolumn{3}{|c|}{ DIRIGIDO AL PROBLEMA } \\
\hline $\begin{array}{l}\text { Dirigida al problema } \\
\text { Directo }\end{array}$ & 5,8 & & \\
\hline $\begin{array}{l}\text { Dirigida al problema } \\
\text { Revaluación } \\
\text { cognitiva positiva }\end{array}$ & 12,9 & & \\
\hline $\begin{array}{l}\text { Dirigida al problema } \\
\text { Revaluación } \\
\text { cognitiva negativa }\end{array}$ & 15,10 & & \\
\hline
\end{tabular}

\begin{tabular}{|c|c|c|c|c|c|}
\hline PERCENTIL & $\begin{array}{c}\text { DIRIGIDO } \\
\text { A LA } \\
\text { EMOCIÓN } \\
\text { Evitativo }\end{array}$ & $\begin{array}{c}\text { DIRIGIDO } \\
\text { A LA } \\
\text { EMOCIÓN } \\
\text { Emocional } \\
\text { negativo }\end{array}$ & $\begin{array}{c}\text { DIRIGIDO } \\
\text { AL } \\
\text { PROBLEMA } \\
\text { Directo }\end{array}$ & $\begin{array}{c}\text { DIRIGIDO } \\
\text { AL } \\
\text { PROBLEMA } \\
\text { Revalorativo } \\
\text { Positivo }\end{array}$ & $\begin{array}{c}\text { DIRIGIDO } \\
\text { AL } \\
\text { PROBLEMA } \\
\text { Revalorativo } \\
\text { Negativo }\end{array}$ \\
\hline 25 & 2 & 1 & 3 & 2 & 1 \\
\hline 50 & 4 & 4 & 5 & 4 & 4 \\
\hline 75 & 6 & 6 & 6 & 6 & 6 \\
\hline
\end{tabular}

